\title{
Erratum to: High-frequency diffraction of an electromagnetic plane wave by an imperfectly conducting rectangular cylinder
}

\author{
Anthony D. Rawlins
}

Received: 26 May 2014 / Accepted: 26 May 2014 / Published online: 2 October 2014

(C) Springer Science+Business Media Dordrecht 2014

Abstract In this note we correct some typographical errors in the above paper.

Keyword Diffraction Theory

Mathematics Subject Classification 3C

Erratum to: J Eng Math (2012) 76:157-180

DOI 10.1007/s10665-011-9516-y

In Eqs. (40) to (43), $\cos \left[4 \theta_{0} / 3\right]$ should be replaced by $\cos \left[4\left(\theta_{0}-\pi / 2\right) / 3\right]$; and in Eq. (44), $\cos \left[4 \theta_{0} / 3\right]$ should be replaced by $\cos \left[4\left(\theta_{0}-\pi\right) / 3\right]$.

These corrections do not affect any subsequent formulae appearing in the paper. In carrying out these corrections in the software, for the range of parameters that were used, no significantly discernable changes occurred to the graphs given in Figs. 20-25 already presented. I would also like to make clear that the expressions in my paper are not uniformly valid across the angular rays $\theta=0, \pi / 2, \pi, 3 \pi / 2,2 \pi$. This is because the derivatives of the diffraction coefficients become infinite along these grazing incidence directions. In the plots presented in the paper interpolation was carried out around these angular directions. I would like to thank Professor Paul Smith of The Mathematics Department, Macquarie University, Sydney, Australia for alerting me to these shortcomings after he ran an accurate numerical check by an integral equation method.

The online version of the original article can be found under doi:10.1007/s10665-011-9516-y.

A. D. Rawlins $(\bowtie)$

Department of Mathematical Sciences, Brunel University, Uxbridge, Middlesex UB8 3PH, UK

e-mail: mastadr@brunel.ac.uk 